\title{
artigo
}

Lima, A.S.G.; Ribeiro, K.C.S.; Sousa, V.V.S.; Barbosa, A.L.; Lima, R.S.B.; Rodrigues, A.R.M.

A utilização de álcool na população adolescente e sua relação com ideias suicídas

DOI: https://doi.org/10.36489/saudecoletiva.2020v10i58p4153-4066

\section{A utilização de álcool na população adolescente e sua relação com ideias suicídas}

The use of alcohol in the adolescent population and its relationship with suicide ideas

El uso de alcohol en la población adolescente y su relación con las ideas del suicidio

\section{RESUMO}

Objetivo: Esta pesquisa teve como objetivo analisar a relação entre o consumo de álcool em adolescentes e sua relação com o suicidio em escolas estaduais de João Pessoa. Método:Para a realização do estudo utilizou-se um questionário autoaplicável que abordavam questões referentes ao consumo de bebida e ideações suicidas. Os dados foram analisados por estatística descritiva e Qui-quadrado. A amostra foi composta por 1142 estudantes com média da idade de 16,38 anos. Resultados: podemos destacar que $12 \%$ da população da amostra planejaram suicidar-se e 7,4\% tentaram suicidar-se. Acrescentando com consumo de álcool, onde cerca de 40,9\% ingeriram bebida alcoólica no último mês. As análises demonstraram a existência de uma relação entre o plano para o suicídio vinculado a quantidade de bebida no último mês (x2 20,116; p<0,000), como também a Tentativa do suicídio vinculado com a quantidade bebida no último mês (x2 17,126; p<0,017). Conclusão: portanto, indicar possíveis estratégias para o desenvolvimento de intervenções eficazes neste grupo etário.

DESCRITORES: Álcool; Adolescentes; Suicídio.

\section{ABSTRACT}

Objective: This research aimed to analyze the relationship between alcohol consumption in adolescents and its relationship with suicide in state schools in João Pessoa. Method: To carry out the study, a self-administered questionnaire was used, which addressed issues related to drinking and suicidal ideas. The data were analyzed using descriptive statistics and Chi-square. The sample consisted of 1142 students with an average age of 16.38 years. Results: we can highlight that $12 \%$ of the sample population planned to commit suicide and $7.4 \%$ tried to commit suicide. Adding to alcohol consumption, where about $40.9 \%$ had drunk alcohol in the last month. The analyzes showed the existence of a relationship between the plan for suicide linked to the amount of drink in the last month (x2 20,116; $<$ <.000), as well as the attempted suicide linked to the amount of drink in the last month (x2 17.126; $\mathrm{p}<0.017)$. Conclusion: therefore, to indicate possible strategies for the development of effective interventions in this age group.

DESCRIPTORS: Alcohol; Teenagers; Suicide.

\section{RESUMEN}

Objetivo: Esta investigación tuvo como objetivo analizar la relación entre el consumo de alcohol en adolescentes y su relación con el suicidio en las escuelas públicas de João Pessoa. Método: Para la realización del estudio se utilizó un cuestionario autoadministrado, que abordó temas relacionados con la bebida y las ideas suicidas. Los datos se analizaron mediante estadística descriptiva y Chi-cuadrado. La muestra estuvo conformada por 1142 estudiantes con una edad promedio de 16.38 años. Resultados: podemos destacar que el $12 \%$ de la población de la muestra planeaba suicidarse y el $7.4 \%$ intentaba suicidarse. Agregando al consumo de alcohol, donde alrededor del 40,9\% había bebido alcohol en el último mes. Los análisis mostraron la existencia de una relación entre el plan de suicidio vinculado a la cantidad de bebida en el último mes (x2 20,116; p <0,000), así como el Intento de suicidio vinculado a la cantidad de bebida en el último mes (x2 17,126; $p<0,017)$. Conclusion: por tanto, señalar posibles estrategias para el desarrollo de intervenciones efectivas en este grupo de edad.

DESCRIPTORES: Alcohol; Adolescentes; Suicidio.

RECEBIDO EM: 15/09/2020 APROVADO EM: 22/10/2020

\section{Arthur da Silva Gouveia Lima}

Graduado em Psicologia pela Uninassau - Campina Grande - PB.

ORCID: 0000-0003-1190-4892 
Karla Carolina Silveira Ribeiro

Doutora em Psicologia Social pela UFPB - João Pessoa - PB.

ORCID: 0000-0001-7967-5681

Viviane Virginia Silva de Sousa

Graduada em Psicologia pela UEPB - Campinha Grande - PB.

ORCID: 0000-0003-3721-8620

Amanda Laurentino Barbosa

Graduada em Psicologia pela Uninassau - Campina Grande- PB.

ORCID: 0000-0003-1463-3864

Renan da Silva Bezerra de Lima

Graduada em Psicologia pela Uninassau - Campina Grande- PB.

ORCID: 0000-0002-9105-3292

\section{Alanne Renaly Mota Rodrigues}

Graduada em Psicologia pela Uninassau - Campina Grande - PB.

ORCID: 0000-0003-0951-3219

\section{INTRODUÇÃO}

A adolescência é o período de transição, no qual os adolescentes desenvolvem as suas capacidades experimentando novos tipos de comportamento e enfrentam o desafio de adotarem comportamentos saudáveis ${ }^{1}$. Corroborando, essas afirmações ${ }^{2}$ referem-se à adolescência como uma fase marcada por grandes descobertas e instabilidade emocional, período no qual é consubstanciada a personalidade. Essa fase não pode ser reduzida a uma simples faixa etária, pois tratam-se da transformação para a vida adulta e, portanto, de fase de decisões biológicas, sociais e, principalmente, psicológicas para toda a vida.

Contudo, a fase de desenvolvimento humano associado a adolescência dentro dos recortes legais passa a ser descritos em faixas etárias, sendo que para Organização Mundial da Saúde $(\mathrm{OMS})^{3}$ a idade cronológica de um adolescente está definida entre seus 10 a 19 anos, já o Estatuto da Criança e do Adolescente (ECA)4 no Brasil, Lei 8.069, de 1990, conceito este utilizado no presente trabalho, traz outra vertente, definindo uma faixa etária entre os 12 e 18 anos.

$\mathrm{O}$ adolescente experimenta uma autonomia de valores e opiniões, encontrando um leque de alternativas laborais e galgar a possibilidade de inserção entre grupos formais ou informais, para tanto passa a ado- tar o perfil social dos seus pares, associando comportamentos a liberdade e aceitação, entre eles o destaque ocorre no álcool, tabaco e iniciação sexual's. Dessa forma os adolescentes preocupam-se com os papéis sociais que desempenharão em suas vidas, projetando planos referentes a hobbies, estudos, profissão, grupos e outros fatores que contribuem para a construção da identidade ${ }^{6}$ Assim, esse período de passagem, o jovem começa a imitar uma série de comportamentos dos adultos e, entre eles, o que será destaque neste trabalho é o consumo o do álcool e suas consequências.

Os problemas emocionais enfrentados pelos jovens representam uma das causas do alto consumo de álcool. Um sujeito que esteja passando por momento de tensão, conflitos, problemas nos relacionamentos familiares ou afetivos pode consumir álcool para combater a depressão e os sentimentos indesejados. No entanto, o consumo tem efeito depressivo e só pode agravar os problemas, levando os mesmos a apresentarem uma frequência maior de pensamentos mórbidos ${ }^{7}$.

Frente ao exposto e compreendendo que a puberdade é marcada por inconstâncias emocionais e inseguras sociais, devido a necessidade de aceitação desses sujeitos em desenvolvimento por seus pares, o que pode acarretar o maior consumo de bebida alcoólica ${ }^{7}$. Entende-se que a população de adolescentes é exposta à vulnerabilidade social e que a vulnerabilidade pode ser caracterizada como a exposição das pessoas ao adoecimento ${ }^{8}$.

Nesta perspectiva, traz a ideia de vulnerabilidade não ser apenas de forma individual, mas de uma forma lógica que está presente nas diversas relações econômicas, sociais, culturais e políticas da sociedade. Enquanto os fatores de risco indicam probabilidades, a vulnerabilidade é um indicador da iniquidade e da desigualdade social. Desta forma, a vulnerabilidade antecede o risco e determina processos diferenciados de saúde e doença e as possibilidades para o seu enfrentamento, visto que essa vulnerabilidade faz com o que aumente o nível de ideais mórbidos e a prevalência do suicídio?.

Suicídio é uma palavra originada no latim, derivada da junção das expressões sui (si mesmo) e caederes (ação de matar). Em outras palavras significa o ato voluntário do indivíduo e o seu desejo de tirar a própria vida $^{10}$. Frente à realidade dos adolescentes e sua relação com o suicídio, uma pesquisa que foi realizada pelo Diário do Nordeste em 201711 mostra que uso de álcool por adolescentes aumenta o risco de suicídio e ainda mais, a experimentação de bebidas alcoólicas por adolescentes com idade entre 13 a 15 anos subiu de 50,3\% em 2012 para 55,5\% em 2015. Nessa mesma pesquisa temos alguns dados relevantes do Instituto Brasileiro 


\section{artigo}

de Geografia Estatística (IBGE)12 que definiu pelos Dados da Pesquisa Nacional de Saúde do Escolar (PeNSE) de 2015 os alunos entre 13 a 15 anos, a experimentação de álcool subiu de 50,3\% em 2012 para 55,5\% em 2015. Além disso, 21,4\% desses adolescentes relataram já terem sofrido algum episódio de embriaguez na vida.

De acordo com a OMS $(2015)^{13}$, a restrição ao acesso aos meios de cometer suicídio, a identificação e o tratamento precoce de pessoas que sofrem de transtornos psicológicos, especialmente a depressão, bem como daquelas que abusam de substâncias, o aperfeiçoamento do acesso aos serviços sociais e de saúde são estratégias efetivas para a prevenção. Corroborando com essa afirmação, Masur e Monteiro ${ }^{14}$ discorrem que o consumo de álcool pode ser resultante do estilo de vida atual, dos altos níveis de estresse, de baixa autoestima, sentimentos depressivos, de ansiedade, susceptibilidade à pressão dos pares e problemas relacionados à escola. Visto que, o uso exagerado e indevido de bebidas alcoólicas é considerado um grave problema de saúde pública.

Nesta perspectiva, Reis, Húngaro e Oliveira ${ }^{15}$ relatam a importância sobre os estudos sobre o tema abordado, trazendo questôes sobre a necessidade de mais programas interventivos que tenha uma eficácia mais ampla nos resultados de relação entre o álcool e suicídio, pois estudo do Ministério da Saúde, a cartilha da OMS13 e informações contidas na revista A Tribuna (2016) ${ }^{16}$ articulam-se mostrando que nos últimos cinco anos, houve um crescente aumento de suicídios e que hoje é a segunda maior causa de morte em jovens.

Considerando a importância de estudos acima que tiveram grande relevância na utilização de álcool em adolescentes, buscando a vulnerabilidade como fatores de risco, e sua relação com ideias mórbidas, esse trabalho objetivou analisar a relação entre o consumo de álcool em adolescentes e a ideia suicida, que deve contribuir na produção de resultados e, indicando possíveis estratégias para o desenvolvimento de intervenções eficazes para esta e outras amostras futuras.

\section{MÉTODO}

$\mathrm{O}$ presente estudo caracterizou-se como uma pesquisa aplicada, de abordagem transversal quantitativa, com o objetivo de estudo descritivo e exploratório, com procedimentos técnicos de levantamento a que se refere se o uso do álcool tem influência significativa para a ideação suicida dentre os seguintes quesitos: pensamento, planejamento e tentativa.

Participaram desta pesquisa 1142 estudantes da rede estadual de ensino na cidade de João Pessoa - PB dentre o período de setembro e outubro. A população desde estudo foi constituída por adolescentes de 12 a 18 anos, do sexo masculino e feminino, matriculados em escolas estaduais da região de João Pessoa - PB.

Foi utilizado um questionário autoaplicável com questões biodemográficas e relacionadas ao uso de álcool, idade da primeira experimentação e motivação para o uso, bem como ao suicídio (ideação, planejamento e tentativa suicida), construído com base nos estudos de "The Behavioral Risk Factor Surveillance System" (BRFSS) 17, 18, 19,20.

A pesquisa foi aprovada pelo Comitê de Ética em Pesquisa do Centro de Ciências da Saúde da Universidade Federal da Paraíba e autorizada pela Secretaria Estadual de Educação, buscando respeitar todos os aspectos éticos que tratam da pesquisa com seres humanos. A aplicação do questionário foi realizada por pesquisadores devidamente treinados e se deu de forma coletiva em ambiente de sala de aula.
Os dados foram digitados na forma de banco de dados do software para Windows, Statistical Package for Social Science - SPSS e efetuada as análises descritivas (Medidas de Posição e Variabilidade) para delinear o perfil sociodemográfico da amostra. Na sequência, utilizamos a estatística Não-Paramétrica (Qui-Quadrado) e testes bivariados a fim de verificar a existência ou não de associações entre as variáveis da pesquisa.

A pesquisa foi aceita e aprovada pelo comitê de ética com o CAAE: 95101418.0.0000.5193 e com comprovante de número $087015 / 2018$ obedecendo Resolução $n^{\circ} 466^{21}$, de 12 de dezembro de 2012.

\section{RESULTADOS}

A média de idade entre os estudantes foi de 16,38 anos ( $D P=1,448)$ onde foi caracterizada por alunos matriculados no ensino médio, quanto às informaçóes sociodemográficas, $42.1 \%$ são do sexo masculino e $57,9 \%$ do sexo feminino. Segue abaixo, Tabela 1 com as descrições sociodemográficas dos participantes:

No que diz respeito à faixa etária, é notório que a maioria dos adolescentes tinha como faixa etária entre os 15 e 16 anos, com $47,5 \%$, logo após com $36,1 \%$ da amostra, alunos entre 17 e 18 anos, em seguida com $8,8 \%$ alunos entre 12 e 14 anos e por fim, alunos entre 18 e 19 anos com 7,6\%, no qual esses alunos tinha uma significância maior no

\section{Tabela 1- Caracterização sociodemográfica da amostra}

\begin{tabular}{|c|c|c|c|}
\hline C & Niveis & $F$ & $\%$ \\
\hline \multirow[t]{4}{*}{ Faixa etária } & $12-14$ & 101 & 8,8 \\
\hline & $15-16$ & 542 & 47,5 \\
\hline & $17-18$ & 412 & 36,1 \\
\hline & $18-19$ & 087 & 7,6 \\
\hline \multirow[t]{3}{*}{ Série Escolar } & Primeiro Ano & 453 & 39,7 \\
\hline & Segundo Ano & 414 & 36,3 \\
\hline & Terceiro Ano & 275 & 24,1 \\
\hline \multirow[t]{2}{*}{ Sexo } & Masculino & 481 & 42,1 \\
\hline & Feminino & 661 & 57,9 \\
\hline
\end{tabular}


primeiro ano de ensino médio com 39,7\%, $36,3 \%$ no segundo e $24,1 \%$ no primeiro. É importante salientar sobre a pesquisa que foi realizada com alunos entre 12 a 18 anos, onde a maior frequência se dá entre alunos menores de idade, definindo assim um grave problema de conscientização e ainda ressalva a lei 243 do ECA (1990)4 que é terminantemente proibido a venda de bebida alcoólica para menores de 18 anos, o que causa mais insegurança e maior vulnerabilidade de desenvolver problemas de saúde na vida adulta, podendo destacar o alcoolismo.

Objetivando-se analisar a prevalência da utilização de álcool em adolescentes, foram realizadas algumas médias de variáveis, sobre a primeira vez em que o adolescente ingeriu álcool, a motivação para a utilização e a frequência de vezes o qual o mesmo usou no último mês. A média de idade dos participantes para a primeira experimentação do álcool foi de 13,84 anos $(\mathrm{DP}=1,997)$. Abaixo, segue tabela que caracteriza as variáveis explanadas anteriormente:

Segundo a Tabela 2, é possível verificar que $76,3 \%$ dos adolescentes já consumiram algum tipo de bebida alcoólica, desses 40,9\% fizeram consumo no último mês, ou seja, o álcool está presente na vida cotidiana desses estudantes.
Neves22 relata que a droga mais utilizada pelos adolescentes é o álcool e ainda acrescenta que existem fatores motivacionais para o consumo da mesma. A oferta e acessibilidade do uso da bebida alcóolica, pode direcionar esses estudantes a um consumo precoce gerando uma ampla disponibilidade do álcool nos ambientes, os deixando em situações de vulnerabilidade e predisposição ao vício23. Corroborando com esses dados, Ayres 24 retrata que o trabalho em que a vulnerabilidade desenvolve em totalidades compreensivas, onde nas questões de exposições a infecção e adoecimento são referidos os aspectos individuais, sociais e programáticos.

Quanto às motivações para o adolescente fazer o uso do álcool, os dados mostram com certa relevância "Simpático, alegre, animado" com 19,1\% e "Aceitação ou influência do grupo de amigos com $13,1 \%$, mostrando assim que essas pessoas são expostas a fatores de riscos, são vulneráveis ao consumo. Geralmente é onde os adolescentes começam a mudar fisicamente, psicologicamente e socialmente, assim mudando relativamente os comportamentos e adaptando-se a novos comportamentos. Corroborando com esses dados, Cavalcante, Alves e Barroso2 retrata que o uso de álcool traz uma segurança ao

\section{Tabela 2- Consumo de Álcool}

\begin{tabular}{lccc}
\multicolumn{1}{c}{ Variáveis } & Niveis & F & $\%$ \\
Já consumiu bebida alcoólica? & Sim & 871 & 76,3 \\
Quantidade de bebida no & Não & 270 & 23,7 \\
último mês? & nenhuma vez & 517 & 59,2 \\
& 1 - 5 vezes & 269 & 30,8 \\
Motivação para a Bebida & 6es ou mais vezes & 88 & 10,1 \\
& Desibição e ajuda relacionamento & 58 & 7,8 \\
& Social & 08 & 1,1 \\
& Relações sexuais & 142 & 19,1 \\
& Simpático alegre animado & 85 & 11,5 \\
& Esquecer coisas ruins & 27 & 3,6 \\
& Faz tudo parecer fácil & 97 & 13,1
\end{tabular}

convívio com os amigos, por falta de maturidade, alguns utilizam por impulso ou até mesmo para serem aceitos em grupos mais influentes, visto que eles são expostos a situações facilitadoras ao consumo precoce da bebida alcoólica.

Nesta perspectiva Papalia25 afirma que no contexto social, alguns fatores permeiam como vulnerabilidade para os adolescentes, visto que os mesmos procuram buscar novas experiências, obtendo prazeres físicos, encontrar alívios de pressões externas, visto que são bastante pressionados, além de ter um ciclo de amizades em que precisem acompanhar o ritmo de atitudes e comportamentos. Corroborando com a afirmação anterior Ribeiro26 explana a ideia da necessidade do adolescente se sentir aceito pelos pares e definir sua identidade, os faz aderir a processos de experimentação, podendo apresentar comportamentos vulneráveis de risco ao álcool e até mesmo a outras drogas.

Um outro fator importante é que, 11,5\% dos adolescentes usam o álcool porque acreditam que "Esquecem coisas ruins", já 3,6\% acreditam que "Faz tudo parecer fácil", como um pensamento de fuga de problemas que acometem o dia a dia desses jovens e isso pode gerar grandes prejuízos para o desenvolvimento dos mesmos. Esses pensamentos possam estar interligados a questôes socioeconômicas, envolvimento com criminalidade, cultura, dificuldade nas relações familiares, rendimento escolar ${ }^{27}$. Atanazio e et al. ${ }^{28}$ corrobora com alerta da utilização precoce do álcool por esses adolescentes, devido ao risco de surgirem consequências graves no futuro, podendo atingir todo o domínio biopsicossocial desses indivíduos. Assim percebe que nestes indivíduos questões de cunho psicoafetivo e dificuldade ambientais, que podem estar atrelados a transtornos como depressão e ansiedade, os faz procurar o comportamento de beber.

O uso de bebida alcoólica com motivação para as "relações sexuais" teve uma frequência $1,1 \%$ dentro da amostra, trazendo para a reflexão que essa população podem desenvolver problemas sérios, caso não façam o uso de preservativos que vai desde a gravidez na adolescência, a contaminação com DSTs (doenças sexualmente transmissíveis) ${ }^{29}$. 


\section{artigo}

Lima, A.S.G.; Ribeiro, K.C.S.; Sousa, V.V.S.; Barbosa, A.L.; Lima, R.S.B.; Rodrigues, A.R.M.

A utilização de álcool na população adolescente e sua relação com ideias suicídas

Como forma de compreender melhor o objetivo do estudo, analisar a relação entre o consumo de álcool em adolescentes e sua relação com a ideia mórbidas, a Tabela 3 , irá apresentar dados referentes aos estudantes que relataram apresentar algum ponto de ideação, ou pensar, planejar ou já ter tentado tirar a própria vida.

Segundo Werlang, Borges, Fensterseifer ${ }^{30}$ o comportamento suicida está interligado de forma isolada ou de forma frequente, sentimentos, desejos e manifestações do propósito de querer morrer, assim engloba o planejamento de como fazer isso, quando e onde vai fazer isso, além do pensamento de como esse "suicídio" pode desenvolver impactos na vida dos outros. Dentre as mesmas podemos avaliar que dentro do pensamento $24,3 \%$ já pensaram em se matar, ou seja, acredita-se que a ideação não estar relacionada apenas o ato, e sim desde o pensamento, e que é necessário não só apenas a detecção precoce desses pensamentos, mas também tudo o que foi feito para que esses pensamentos surgissem ${ }^{31}$.

Diante o planejamento percebe-se que há uma variação decrescente onde confirma dentro da amostra que $12 \%$ já fizeram "Plano para o suicídio" e 7,4\% já praticaram a "Tentativa do suicídio" e conforme estudos o planejamento está diretamente ligado a solução de problemas e a partir daí vem o comportamento da tentativa, ou seja, existe a idealização para encontrar a solução e usa da tentativa como a resolução, isso acontece pela fixação mental no pensamento, através de estereótipos mentais e vieses negativos. Sampaio e Silva ${ }^{32}$ corroboram afirmando que o suicídio na

\section{Tabela 3- Ideação à Ação Mórbida}

$\begin{array}{cccc}\text { C } & \text { Níveis } & \text { F } & \% \\ \text { Pensou em se matar? } & \text { Sim } & 277 & 24,3 \\ \text { Plano para o suicídio? } & \text { Não } & 865 & 75,7 \\ & \text { Sim } & 137 & 12,0 \\ \text { Tentativa de Suicídio? } & \text { Não } & 1005 & 88,0 \\ & \text { Sim } & 85 & 7,4 \\ & \text { Não } & 1057 & 92,6\end{array}$

Tabela 4- Plano para suicídio X Quantidade bebida último mês

Quantidade bebida último mês

Total nenhuma vez $\quad 1-5$ vezes $\begin{gathered}6 \text { ou mais } \\ \text { vezes }\end{gathered}$

$\begin{array}{lrcccc}\text { Plano para suicídio? } & \text { sim } & 20 & 51 & 49 & 120 \\ & \text { não } & 497 & 218 & 39 & 754 \\ \text { Total } & & 517 & 269 & 88 & 874 \\ \text { *Teste qui-quadrado 20,116(p<0,000). } & & & & \end{array}$

Tabela 5- Tentativa suicídio X Quantidade bebida último mês

\begin{tabular}{lccccc} 
& & \multicolumn{2}{c}{ quantidade bebida último mês } & Total \\
& & nenhuma vez & $1-5$ vezes & $\begin{array}{c}\text { 6 ou mais } \\
\text { vezes }\end{array}$ & \\
tentativa suicídio? & sim & 13 & 28 & 34 & 75 \\
& não & 504 & 241 & 54 & 799 \\
Total & 517 & 269 & 88 & 874 \\
*Teste qui-quadrado 17,126(p<0,017) & & & &
\end{tabular}

adolescência é algo que retrata muito peso emocional ao jovem e seus familiares, é gerado um sofrimento psíquico que às vezes pode ser difícil de satisfazer, e também por existirem questionamentos a respeito da situação e do "tempo que foi perdido".

Tendo como objetivo alcançar o que foi planejado no presente estudo, foi realizado um cruzamento entre as Tabelas 2 e 3 (Consumo de Álcool e Ideações suicidas), entre os dados cruzados, só apresentaram resultados significativos: Plano para o suicídio $\mathrm{x}$ quantidade de bebida no último mês e Tentativa para o suicídio $x$ quantidade bebida no último mês. Tendo como propósito identificar a associação entre a quantidade de bebida alcoólica utilizada no último mês pelos adolescentes e plano suicida, foi realizada uma tabulação cruzada (qui-quadrado), para verificar o quantitativamente a relação entre o consumo de álcool e a frequência esperada para o fenômeno (plano de suicídio). Esses mesmos resultados também foram encontrados no cruzamento entre a tentativa de suicídio (ato de se matar) com a frequência de álcool consumido no último mês, Tabela 5, abaixo:

Os dois cruzamentos evidenciaram que quanto maior for o consumo de álcool, maior é a frequência do planejamento, como também do risco do ato, isto a tentativa, o que pode levar a morte desses púberes. Para Wilson e Kalander ${ }^{33}$ os adolescentes são submetidos a situações que provocam risco a saúde, esses fatores promovem consequências que maximizam a dependência, cabe ressaltar o uso de álcool e drogas, Já Ayres 10 traz esses fatores, como fatores de risco que precisam de uma atenção voltada para a prevenção dos mesmos.

\section{DISCUSSÃO}

Podemos confirmar a respeito do estudo realizado que o consumo do álcool está atrelado a fatores emocionais, como está descrito nos níveis às motivações para o uso do álcool, e assim podemos assimilar que dentro da amostra, houve uma grande parte da população que indicava necessidade de esquecer coisas ruins e ter alegria de alguma maneira, e acrescentando tam- 
bém o consumo relaciona-se também pela necessidade de aceitação social, o qual pode delinear um perfil suicida. Pires34 destaca que existem conformidades relacionadas ao suicídio que estão interligadas ao contexto social. Assim podemos usar como ressalve que aspectos motivacionais escolhidos pelos participantes possam ter ligação concomitante com o uso de bebida alcoólica e o suicídio.

Assim, observa-se uma necessidade ampla de avaliar as questóes motivacionais para compreender o que levam essa população ao consumo da bebida alcoólica, pois pelos dados aqui apresentados, existe uma maior frequência deste comportamento atrelado ao planejamento e comportamento suicida. Assim, oferecer um atendimento especializado em saúde como forma de prevenção e atenção a essa população para minimizar o índice de comportamento extremistas frente a vida.

\section{CONCLUSÃO}

Diante este estudo, conclui-se que a partir dos resultados alcançados, é possível identificar que a vulnerabilidade é um fator de risco que o adolescente acomete, ou seja, o adolescente é predisposto a situações expostas na sociedade. O uso de álcool pelos mesmos pode trazer um benefício a nível curto prazo, que abstém os resultados da pesquisa, evidenciando-se o sentir-se "simpático, alegre, animado" como fator motivacional para o consumo da droga. Vale ressaltar que a aquisição do álcool é algo muito acessível, mesmo sabendo que existem leis que proíbam a venda para os mesmos e que Schlosser35 corrobora afirmando que o período da adolescência é considerado de maior vulnerabilidade aos comportamentos suicidas.

Assim podemos confirmar a hipótese de que existe correlação entre o uso abusivo do álcool e pensamentos suicidas entre os adolescentes e o problema de pesquisa que seriam os fatores de risco que estão associados ao uso de álcool em adolescentes, que podemos destacar "Aceitação no grupo" e "Esquecer coisas ruins" e sua relação com ideias suicidas que vai desde o pensamento em se matar até o ato em si.

A pesquisa ainda procurou fazer jus à importância que o tema pode acrescentar em estudos da área. Contribuindo à literatura sobre adolescência, utilização do álcool e ideação suicida. Considerando a importância do uso de álcool como fuga psíquica e da ideação suicida no que tange aos riscos que tornam os adolescentes vulneráveis a tal relação, faz-se necessárias intervenções de cunho exploratório que busque o bem-estar dos mesmos, visto que essas práticas acarretam o agravo de doenças psicossomáticas dentro do contexto social, econômico, familiar e educacional.

\section{REFERÊNCIAS}

1.Newman K, Harrison L, Dashiff C, Davies S. Relações entre modelos de pais e comportamentos de risco na saúde do adolescente: uma revisão integrativa da literatura. Rev. Latino-Am. Enfermagem [Internet]. 2008 Feb [cited 2018 Oct 03] ; 16( 1 ): 142150. Available from: http://www.scielo.br/scielo.php?script=sci arttext\&pid=S0104-11692008000100022\&Ing=en. https://doi. org/10.1590/S0104-11692008000100022.

2.Cavalcante MBPT, Alves MDS, Barroso MGT. Adolescência, álcool e drogas: uma revisão na perspectiva da promoção da saúde. Esc. Anna Nery [Internet]. 2008 Set [citado 2018 Out 03] ; 12( 3 ): 555-559. Disponivel em: http://www.scielo.br/scielo.php?script=sci_arttext\&pid=S1414-81452008000300024\&lng=pt. https://doi. org/10.1590/S1414-81452008000300024.

3.Organização Mundial da Saúde [OMS]. Strengthening the health sector response to adolescent health and development. 2010. Acesso em: 03 de março de 2020. Disponivel em: https://www. who.int/maternal_child_adolescent/documents/cah_adh_flyer_2010_12_en.pdf?ua=1

4.Brasil. Lei $n^{\circ}$ 13.257, de 8 de março de 2016. Dispõe sobre o Estatuto da Criança e do Adolescente e dá outras providências. Estatuto da Criança e do Adolescente (ECA). Diário Oficial da União. De março de 2016. Disponivel em: http://www.planalto.gov.br/ccivil_03/LEIS/L8069.htm

5.Aquino IB. Os impactos do uso excessivo de álcool na adolescência. [publicação na web\}; 2014. Acesso em: 30 de abril de 2018. Disponível em http://www.diaadiaeducacao.pr.gov.br/portals/cadernospde/pdebusca/producoes_pde/2013/2013_unicentro_bio_ pdp_irene_barros_de_aquino.pdf

6.Campos DMS. Psicologia da adolescência: normalidade e psicopatologia. Rio de Janeiro: Vozes; 2012.

7.Teixeira PS, Stefanini MCB, Martins RA, Cruz LAN da. Desenvolvimento cognitivo e sintomas depressivos em adolescentes que fazem uso de bebidas alcoólicas. SMAD Rev Eletr Saúde Mental Álcool Drog [Internet]. $1^{\circ}$ de abril de 2011 [citado $3^{\circ}$ de outubro de 2018];7(1):03-9. Disponível em: http://www.revistas.usp.br/smad/ article/view/38733

8.Brêtas JRS. Vulnerabilidade e adolescência. Revista da Sociedade. Revista da Sociedade Brasileira de Enfermeiros Pediatras. 2010; 10(2), 89-96. Acesso em: 03 de março de 2020. Disponível em: https://sobep.org.br/revista/images/stories/pdf-revista/vol10-n2/ v.10_n.2-art5.refl-vulnerabilidade-e-adolescencia.pdf

9.Ayres, J. R. Sobre o risco: para compreender a epidemiologia. São Paulo: Hucitec; 2002.

10.Vieira, KFL. Depressão e suicídio: uma abordagem psicossociológica no contexto acadêmico. 2008. João Pessoa: Universidade Federal da Paraíba. Dissertação de mestrado.

11.Diário do Nordeste. Uso de álcool por adolescentes aumenta risco de suicídio. 2017. Acesso em: 03 de outubro de 2018. Disponível em: http://diariodonordeste.verdesmares.com.br/editorias/verso/ online/uso-de-alcool-por-adolescentes-aumenta-risco-de-suicidio-1.1815281

12.Instituto Brasileiro de Geografia Estatística (IBGE) [base de dados online]. Rio de janeiro: Pesquisa nacional de saúde do escolar 


\section{REFERÊNCIAS}

(PeNSE). 2015. Acesso em: 14 de junho de 2018. Disponível em: https://biblioteca.ibge.gov.br/visualizacao/livros/liv97870.pdf

13.Organização Mundial da Saúde [OMS]. Adolescent health research priorities: report of a Technical consultation. 2015. Acesso em: 03 de março de 2020. Disponivel em: http://apps.who.int/iris/bitstream/handle/10665/203564/WHO_FWC_MCA_15_07_eng. pdf; ;sessionid=48A79DDFB0C40E4262C4DBEE386AD3EE?sequence $=1$

14.Masur, J, Monteiro MG. Validation of the CAGE alcoholism screening test in a Brazilian psychiatric inpatient hospital setting. Brazil Journal Med Biol Res, v. 16, p. 215-8, 1983.

15. Reis LM, Hungaro AA, Oliveira MLF. Políticas públicas para o enfrentamento do uso de drogas de abuso: percepção social em uma comunidade. Texto contexto - enferm. [Internet]. 2014 Dec [cited 2018 Jun 03] ; 23( 4 ): 1050-1058. Available from: http://www.scielo.br/scielo.php?script=sci_arttext\&pid=S0104-07072014000401050\&Ing=en. https://doi. org/10.1590/0104-07072014002620013.

16.A Tribuna. Suicídio é a segunda maior causa de morte entre os jovens. 2016. Acesso em: 03 de outubro de 2018. Disponível em: http://www.atribuna.com.br/noticias/noticias-detalhe/cidades/ suicidio-e-a-segunda-maior-causa-de-morte-entre-os jovens/?cHash=d49c717862a27c7e9a6bf852a1f5a24c

17.United States Department of Heath and Human Services [USDHHS]. The Behavioral Risk Factor Surveillance System (BRFSS). 1999. Acesso em: 03 de março de 2020. Disponível em: https:// www.cdc.gov/brfss/annual_data/1999/pdf/overview_99.pdf

18.Farias Jr. JC. Estilo de vida de escolares do ensino médio no município de Florianópolis, Santa Catarina, Brasil. Santa Catarina: Universidade Federal de Santa Catarina, 2002. Dissertação de mestrado.

19.De Bem, MFL. Estilo de vida e comportamentos de risco de estudantes trabalhadores do ensino médio de Santa Catarina. Santa Catarina: Universidade Federal de Santa Catarina, 2003. Dissertação de doutorado.

20.Azevedo, RLW. Aspectos psicossociais da sexualidade adolescentes associada à vulnerabilidade ao HIV/AIDS. João Pessoa: Universidade Federal da Paraíba, 2007. Dissertação de mestrado.

21.BRASIL. Ministério da Saúde. Conselho Nacional de Saúde. Resolução n 466, de 12 de dezembro de 2012. Diário Oficial [da] República Federativa do Brasil, Brasilia, DF, 12 dez. 2012.

22.Amaral, ACG. O uso do álcool e a vulnerabilidade à aids: estudo com adolescentes gaúchos e paraibanos. João Pessoa: Universidade federal da Paraíba, 2007. Dissertação de mestrado.

23.Neves KC, Teixeira MLO, Ferreira MA. Fatores e motivação para o connsumo de bebidas alcoólicas na adolescência. Esc. Anna Nery [Internet]. 2015; 19(2):286-291. Acesso em: 03 de março de 2020. Disponível em: https://www.scielo.br/pdf/ean/v19n2/1414-8145ean-19-02-0286.pdf.

24.Duailibi, S., \& Laranjeira, R. Políticas públicas relacionadas às bebidas alcoólicas. Revista de Saúde Pública, 2007; 41(5), 839-848. dói:10.1590/S0034-89102007000500019.
25.Ayres JR, Buchalla CM, \& Paiva V. Vulnerabilidade e Direitos Humanos - Prevenção e Promoção da Saúde. Curitiba: Juará; 2012.

26.Papalia DE, \& Olds, S. W. Desenvolvimento humano. $12^{\circ}$ ed. São Paulo: Artmed; 2013.

27.RIBEIRO KCS. Intervenção psicoeducativa dirigida à prevenção de DSTs e gravidez não planejada para adolescentes jovens. 2013. 230 f. João Pessoa: Universidade Federal da Paraíba. Tese (Doutorado em Psicologia Social).

28.Zeitoune RCG, Ferreira VS, Silveira HS, Domingos AM, Maia AC. O conhecimento de adolescentes sobre drogas lícitas e ilícitas: uma contribuição para a enfermagem comunitária. Esc. Anna Nery [Internet]. 2012 Mar [cited 2018 Oct 10] ; 16(1): 57-63. Available from: http://www.scielo.br/scielo.php?script=sci_arttext\&pid=S1414-81452012000100008\&lng=en. https://doi. org/10.1590/S1414-81452012000100008.

29.Atanázio EA, Santos JM, Dionísio LR, Silva J \& Saldanha AAW. Vulnerabilidade ao uso do álcool: um estudo com adolescentes das redes pública e privada de ensino. Abril de 2013. Revista eletrônica saúde mental álcool e drogas, 9(1), 11-17. Acesso em: 10 de junho de 2018. Disponível em: http://pepsic.bvsalud.org/scielo.php?script=sci_arttext\&pid=S1806-69762013000100003\&l$\mathrm{ng}=\mathrm{pt} \&$ tlng=pt.

30.Almeida RAAS, Corrêa RGCF, Rolim ILTP, Hora JM, Linard AG, Coutinho NPS, \& Oliveira PS. Knowledge of adolescents regarding sexually transmitted infections and pregnancy. REBEn. 2017; 70(5): 1033-1039.

31.Werlang SG, Borges VR, Fensterseifer L. Fatores de risco ou proteção para a presença de ideação suicida na adolescência. 2005. Interam J Psychol, 39, 259-66. Acesso em: 06 de jun. de 2018. Disponivel em: http://www.redalyc.org/html/284/28439210/

32.Araújo LC, Vieira KFL, \& Coutinho MPL. Ideação suicida na adolescência: um enfoque psicossociológico no contexto do ensino médio. Psico-USF, 15(1), 47-57. Abril de 2010. Acesso em: 06 de abril de 2018. Disponivel em: https://dx.doi.org/10.1590/S141382712010000100006

33.Sampaio D, \& Silva M. Antidepressivos e suicídio nos adolescentes. 2011. Acta Med Port, 24, 603-612. Acesso em: 03 de outubro de 2018. Disponivel em: https://www.actamedicaportuguesa. com/revista/index.php/amp/article/download/481/189

34.Wilson R, \& Kolander C. Drug abuse prevention: a school and community partnership. 1997. Kentucky: Jones \& Bartlett Learning.

35.Pires MCC, Raposo MCF, Sougey EB, Bastos FOC, Silva TS, Passos MP. Indicadores de risco para tentativa de suicídio por envenenamento: um estudo caso-controle. J. bras. psiquiatr. [Internet]. 2015 Set [citado 2018 Out 03] ; 64( 3 ): 193-199. Disponivel em: http://www.scielo.br/scielo.php?script=sci_arttext\&pid=S0047-20852015000300193\&lng=pt. https://doi. org/10.1590/0047-2085000000078.

36.Schlösser A, Rosa GFC, \& More CLOO. Revisão: comportamento suicida ao longo do ciclo vital. 2014. Temas em Psicologia, 22(1), 133-145. Acesso em: 06 de junho de 2018. Disponível em: https:/ dx.doi.org/10.9788/TP2014.1-11. 